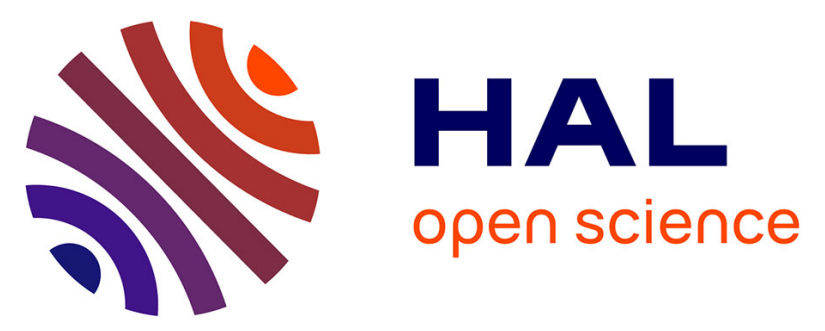

\title{
Validity and sensitivity of the Longitudinal Asymmetry Index to detect gait asymmetry using Microsoft Kinect data
}

Edouard Auvinet, Franck Multon, Victoria Manning, Jean Meunier, Justin P. Cobb

\section{To cite this version:}

Edouard Auvinet, Franck Multon, Victoria Manning, Jean Meunier, Justin P. Cobb. Validity and sensitivity of the Longitudinal Asymmetry Index to detect gait asymmetry using Microsoft Kinect data. Gait \& Posture, 2017, 51, pp.162-168. 10.1016/j.gaitpost.2016.08.022 . hal-01359845

\section{HAL Id: hal-01359845 \\ https://inria.hal.science/hal-01359845}

Submitted on 5 Sep 2016

HAL is a multi-disciplinary open access archive for the deposit and dissemination of scientific research documents, whether they are published or not. The documents may come from teaching and research institutions in France or abroad, or from public or private research centers.
L'archive ouverte pluridisciplinaire HAL, est destinée au dépôt et à la diffusion de documents scientifiques de niveau recherche, publiés ou non, émanant des établissements d'enseignement et de recherche français ou étrangers, des laboratoires publics ou privés. 


\section{Accepted Manuscript}

Title: Validity and sensitivity of the Longitudinal Asymmetry Index to detect gait asymmetry using Microsoft Kinect data

Author: E. Auvinet F. Multon V. Manning J. Meunier J.P. Cobb

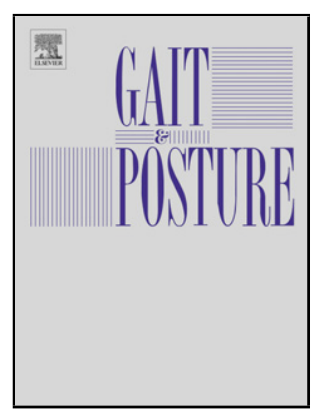

PII: S0966-6362(16)30498-2

DOI: http://dx.doi.org/doi:10.1016/j.gaitpost.2016.08.022

Reference: GAIPOS 5136

To appear in:

Gait \& Posture

Received date: $\quad 1-4-2016$

Revised date: $\quad 17-8-2016$

Accepted date: $\quad$ 22-8-2016

Please cite this article as: Auvinet E, Multon F, Manning V, Meunier J, Cobb J.P.Validity and sensitivity of the Longitudinal Asymmetry Index to detect gait asymmetry using Microsoft Kinect data.Gait and Posture http://dx.doi.org/10.1016/j.gaitpost.2016.08.022

This is a PDF file of an unedited manuscript that has been accepted for publication. As a service to our customers we are providing this early version of the manuscript. The manuscript will undergo copyediting, typesetting, and review of the resulting proof before it is published in its final form. Please note that during the production process errors may be discovered which could affect the content, and all legal disclaimers that apply to the journal pertain. 
Validity and sensitivity of the Longitudinal Asymmetry Index to detect gait asymmetry using Microsoft Kinect data

E Auvinet (1)*, F Multon (2,3), V Manning (1), J Meunier (4), J P Cobb (1)

(1) MSK Lab, Department of Surgery and Cancer, Imperial College London, Charing Cross Hospital, W6 8RP, London, United Kingdom

(2) M2S Laboratory, University Rennes 2, ENS Rennes, Avenue Robert Schuman, 35170 Bruz, France

(3) INRIA, MimeTIC team, Campus Universitaire de Beaulieu, 35042 Rennes, France

(4) Université de Montréal, C.P. 6128, succ. Centre-ville, Montréal H3C 3J7, QC, Canada

* Corresponding author Tel: +44 (0) 203313 4421, email: e.auvinet@imperial.ac.uk 
Research highlights:

- The Continuous Relative Phase is not useable with Kinect.

- New longitudinal index llong is sensitive and validated to detect gait asymmetry.

- The llong index works with Kinect placed either in front of or behind subjects.

- Only few strides (at least 5) are needed to compute a valid Ilong. 


\begin{abstract}
Gait asymmetry information is a key point in disease screening and follow-up. Constant Relative Phase (CRP) has been used to quantify within-stride asymmetry index, which requires noise-free and accurate motion capture, which is difficult to obtain in clinical settings. This study explores a new index, the Longitudinal Asymmetry Index (ILong) which is derived using data from a low-cost depth camera (Kinect). ILong is based on depth images averaged over several gait cycles, rather than derived joint positions or angles. This study aims to evaluate 1) the validity of CRP computed with Kinect, 2) the validity and sensitivity of ILong for measuring gait asymmetry based solely on data provided by a depth camera, 3) the clinical applicability of a posteriorly mounted camera system to avoid occlusion caused by the standard front-fitted treadmill consoles and 4) the number of strides needed to reliably calculate ILong. The gait of 15 subjects was recorded concurrently with a markerbased system (MBS) and Kinect, and asymmetry was artificially reproduced by introducing a $5 \mathrm{~cm}$ sole attached to one foot. CRP computed with Kinect was not reliable. ILong detected this disturbed gait reliably and could be computed from a posteriorly placed Kinect without loss of validity. A minimum of five strides was needed to achieve a correlation coefficient of 0.9 between standard MBS and low-cost depth camera based ILong. ILong provides a clinically pragmatic method for measuring gait asymmetry, with application for improved patient care through enhanced disease, screening, diagnosis and monitoring.
\end{abstract}

Keywords: Kinect; Gait analysis; gait asymmetry; sensitivity analysis; CRP. 


\section{1-Introduction}

Lower-limb gait asymmetry (GA) is characteristic of a number of chronic neurological and musculoskeletal diseases [1], including cerebral palsy [2], Parkinson's disease [3] and knee and hip osteoarthritis [4-5]. Onset of GA can also follow acute events such as stroke [6] and amputation [7]. Thus, quantification of GA has become central to the clinical monitoring and treatment of many chronic and acute neurological and musculoskeletal diseases [8-9].

Gait asymmetry is typically evaluated using kinematic data and spatio-temporal gait parameters (step/stride length and duration). Based on these global variables, indicators have been introduced to quantify asymmetry, including the Symmetry Ratio [6] and Symmetry Index [6]. These indicators provide global asymmetry information for a studied gait cycle, but cannot be used to analyse how asymmetry occurs at specific instances within the gait cycle.

To overcome this limitation, some authors have proposed analysis of the Constant Relative Phase (CRP) and its derivatives [10]. For a given joint, this indicator constructs a phase diagram based on angular value and angular velocity. Gait asymmetry can be assessed by comparing the CRP of the corresponding left and right joints. It has been used to measure asymmetry caused by weights placed on one foot [11] and pathologies such as stroke [12].

However, CRP is based on sagittal joint angles and their derivatives, which impose the use of accurate and noise-free motion capture systems, such as optoelectronic systems $[11,12]$. Such systems are difficult to use in daily practice in small clinics as they require specific technical skills, calibration, and manual processing. In everyday practice, especially in small clinics, easy-to-use and low-cost systems are preferable, such as depth cameras developed for the gaming industries, which have been recently proposed for clinical gait analysis $[13,14]$. However, the authors reported problems when computing temporal information due to inaccuracies and noise. These problems could impose important limitations when computing CRP, especially for the computation of reliable joint angular velocities but this has not yet been tested.

To tackle this potential problem, recent works proposed the direct use of depth images instead of skeleton data to perform clinical gait analysis. Accurate determination of global spatiotemporal data [15] and a continuous asymmetry index - the Longitudinal Asymmetry Index (ILong) [16] has been investigated using data obtained from low cost depth cameras such as the Kinect. ILong evaluates gait asymmetry at each instant of the gait cycle according to the instantaneous spatial distance between the right leg motion expressed during a stride starting with a right-foot strike, and the mirrored left leg motion expressed during a stride starting with a left-foot strike. To reduce noise, a representative gait cycle made of a sequence of averaged depth images (obtained with hundreds of gait cycles) was used to compute ILong. However, no benefit of ILong over CRP was investigated. Moreover, the authors validated this approach with a Kinect placed in front of a treadmill in order to capture and use numerous gait cycles. This was achieved using a specialised treadmill with a remotely placed console so as not to obscure the camera view. ILong computed from an anteriorly placed Kinect would not be applicable to standard treadmills available within a clinical setting, all of which have a front fitting console for safety reasons. 
Therefore, this study aimed to evaluate 1) the validity and sensitivity of the CRP computed with Kinect, 2) validity and sensitivity of ILong for measuring gait asymmetry given the noisy data provided by Kinect, 3 ) the clinical applicability of ILong by placing the Kinect posteriorly rather than anteriorly to the treadmill, to reduce occlusion caused by front-fitted treadmill consoles, and 4) the number of strides needed to reliably calculate ILong.

The main hypotheses were:

- H1: CRP computed with Kinect data is not reliable, supporting the idea of developing a noise-resistant continuous asymmetry index based on Kinect data,

- H2: ILong computed with Kinect and Vicon data is valid and sensitive enough to detect asymmetrical gaits compared to natural ones,

- H3: placing the Kinect behind the subject does not affect the validity of the ILong,

- H4: the number of gait cycles averaged to compute ILong does not strongly affect the results, supporting the idea that a low number of cycles may be possible for some patients.

\section{2-Methods}

A protocol similar to that which has been described previously $[16,17]$ was carried out. Healthy subjects were asked to walk on a treadmill with unilateral imposed perturbation (a $5 \mathrm{~cm}$ sole placed below one of the feet) to induce gait asymmetry. CRP and ILong were then both computed with Kinect and Vicon data concurrently.

\section{1-Participants}

Fifteen healthy subjects (12 males, 3 females, $25 \pm 4$ years old, $172 \pm 7 \mathrm{~cm}$ height and $69.4 \pm 11.6 \mathrm{~kg}$ mass) with no reported clinical asymmetry or gait impairment participated in this study. The University of Montreal ethical review board approved the study and the subjects provided informed consent prior to participation.

\section{2-Protocol}

Participants were asked to perform three walking trials on a treadmill at self-selected comfortable speed [18]: one with normal gait and two with artificially asymmetrical gaits imposed by a $5 \mathrm{~cm}$ sole placed either below the left or the right foot [19]. This artificial way to induce gait asymmetry allowed controlled and reproducible perturbation. The use of a $5 \mathrm{~cm}$ sole was based on previous work which demonstrated that a minimum leg length discrepancy of $4.9 \mathrm{~cm}$ was necessary to generate externally visible effects [19]. The order in which the subject performed the normal and asymmetrical gaits was randomised. Before each trial, the subject had an adaptation time of 2 minutes in order to acclimatise to the artificial deformation. The trial duration was set to 3 minutes to record at least 90 strides.

Gait was measured with both an optoelectronic Vicon (Vicon, Oxford, UK) motion-capture system and two Microsoft Kinect cameras (Microsoft, Redmond, USA): one $2 \mathrm{~m}$ in front, the other $2 \mathrm{~m}$ behind the subject, to evaluate hypothesis H3. Twelve 4-Megapixel infrared Vicon cameras were placed all around the treadmill and recorded the 3D motion of markers placed over standardised anatomical landmarks at $120 \mathrm{~Hz}[15]$.

\section{3-Data analysis}

Gait asymmetry was measured with both CRP and ILong, using both Kinect and Vicon data. 


\subsection{1-Processing of Kinect data}

The joint positions of the skeletons were provided by the Microsoft NUI library (Microsoft, Redmond, USA) and filtered with a zero-phase Butterworth filter with a $7 \mathrm{~Hz}$ cut-off frequency. Stride detection was obtained based on the distance between the knees at a constant estimated knee height [15]. We computed CRP with this Kinect skeleton data on the shank and thigh orientations [12].

ILong was computed using the raw Kinect data (Figure 1) through a four-step process [16]. Firstly, the raw depth images are processed to obtain orthographic projections. Secondly, the recorded data is decomposed into a series of cycles using the gait event detection described above. Thirdly, the method builds a representative cycle of the subject (Mean Gait Cycle Model) by averaging all the compatible depth images in all of the captured gait cycles. Two depth images are considered as compatible if they correspond to similar instances within the gait cycle. For example, all the depth images corresponding to left foot strike events will lead to a unique mean depth image of all left foot heel strikes. This process has previously been successfully applied to 15 successive instances uniformly sampled all along the gait cycle [16]. The last step of this process consists of comparing the depth of the sub-image corresponding to the left leg at time $t$ with the mirrored right leg one at the corresponding time in the cycle. For example, the depth sub-image of the left leg at left heel strike event is compared to the mirrored depth sub-image of the right leg at right heel strike. Hence, perfect symmetrical gait should lead to no difference.

This process was applied to data provided by the Kinects placed in front of and behind the treadmill. To build the Mean Gait Cycle Model, the number of cycles was varied from 1 to 90 to determine the minimum number of cycles required.

\subsection{2-Processing of Vicon data}

ILong and CRP were both computed using Vicon data. Joint centres were computed using the ISB recommendations, and hip joint centre position was computed [20]. The resulting joint positions were filtered with a zero-phase Butterworth filter with a $7 \mathrm{~Hz}$ cut-off frequency [15] similar to the Kinect data. Strides were detected using the same method as applied to Kinect data. Vicon and Kinect data were synchronized using longitudinal distance between legs [15]. CRP at shank and thigh were computed using Vicon data [12]. ILong has been designed for Kinect data, but could also be used with Vicon data [16]. Details about the computation of ILong using Vicon data are given in the supplementary materials. Segments between joint centres were used to artificially generate a depth image and stick figures were used in the remaining process to compute ILong.

\subsection{3-Statistical analysis}

Sensitivity of the various asymmetry indexes and measurement systems were calculated to discriminate the asymmetrical gait from the normal one. Secondly, the method compared the validity of indexes measured with the Kinect in various conditions (placed in front, behind and with various numbers of gait cycles to compute the Mean Gait Cycle Model) to those obtained with the reference Vicon system.

One-way ANOVA tests were conducted at each percent of the gait cycle to examine the significance of the difference between normal and asymmetrical gaits $[16,17]$ with alpha 
level set at 0.05. Tukey-HSD multiple comparison tests were then carried out to identify the differences between the experiments when the null hypothesis was rejected, enabling an evaluation of whether the studied index was able to distinguish if the left and right asymmetrical gaits were significantly different from the normal one.

In order to evaluate the validity of the latter, Pearson correlation coefficients were computed for each reference Vicon index compared with corresponding Kinect indices.

These two statistical tests were applied to:

- $\quad$ CRP computed with both Vicon and Kinect data, testing hypothesis H1,

- ILong computed with both Vicon and Kinect data (using the Kinect placed in front only) using 90 gait cycles to construct the Mean Gait Cycle Model to test hypothesis $\mathrm{H} 2$,

- ILong when placing the Kinect behind the subject, considering 90 gait cycles to construct the Mean Gait Cycle Model to test hypothesis H3,

- ILong considering Mean Gait Cycle Model built using gait cycles 1 to 90. This was applied separately for data given by a Kinect placed in front of or behind the subject to test hypothesis $\mathrm{H} 4$.

\section{3-Results}

Figure 2 depicts CRP computed with Vicon and with Kinect data. As shown in table 1(a), CRP computed with Vicon data was able to detect asymmetry within the stride up to $95 \%$ of the time. However, CRP computed with Kinect data at the shank could only detect asymmetry for $73 \%$ (left deformation) and 52\% (right deformation) within the stride. The results were similar when the CRP was computed at the thigh. Correlation between CRP computed with Vicon and Kinect data all along the cycle were 0.45 and 0.11 at shank and at thigh respectively, demonstrating that noise in Kinect data has a negative impact on the sensitivity and validity of CRP (H1).

Correlation between ILong computed with the Kinect and Vicon data was 0.92 based on 90 averaged cycles and the Kinect placed in front [16] as shown in Figure 3. When using Vicon data, ILong detected that $96 \%$ of the gait cycle exhibits asymmetry, which was similar to results obtained with the Kinect $(91 \%$ and $87 \%$ for the left and right asymmetrical gaits respectively), as shown in table $1(\mathrm{~b})$. This suggests that noise in Kinect data has a low impact on the sensitivity and validity of ILong ( $\mathrm{H} 2)$.

Correlation between CRP and ILong computed with Vicon data was $0.09+-0.43$ for CRP at shank and $0.04+-0.50$ for CRP at thigh, supporting the idea that these two quantities do not measure the same information. They both aim at measuring asymmetry, but CRP describes coordination (joint angle and velocity) within a specific joint for both body sides, whereas ILong measures the geometric difference between the two body sides.

Results obtained with the Kinect placed behind the subject were very similar to those obtained when the sensor was placed in front, table 1 (b). For left asymmetrical gaits, $96 \%$ of the stride was detected as asymmetrical when the Kinect was placed behind, compared to 91\% and $96 \%$ for the Kinect in front and for Vicon data respectively. For right asymmetrical gaits, similar results were obtained: correlation between ILong values computed with Kinect and Vicon data was 0.92 when the Kinect was placed in front and 0.96 when placed behind. 
This suggests that sensor placement has low impact on the sensitivity and validity of ILong $(\mathrm{H} 3)$, and in fact positioning of the Kinect behind the subject may improve accuracy of results.

The close correlation between ILong computed with Kinect and Vicon data is shown in Figure 3. In the two conditions (Kinect in front or behind) with a Mean Gait Cycle Model based on 1-90 cycles, the two techniques agree closely. Correlation for both conditions was greater than 0.85 , even when using a single stride. After just 5 cycles this rapidly grows to a stable value ( 0.89 and 0.93 for placement front and behind respectively). Figure 4 shows the sensitivity of ILong within the gait cycle when using 1, 5 or 90 cycles to build the Mean Gait Cycle Model. It tends to demonstrate that using only a few cycles, even a unique one, did not strongly affect the sensitivity or validity of ILong ( $\mathrm{H} 4)$.

\section{4-Discussion}

The poor results obtained with the CRP computed with Kinect data supports hypothesis $\mathrm{H} 1$ : CRP was not reliably computed from gait data and this could be explained by two reasons. Firstly, the derivation of noisy joint angles delivered by the Kinect induces noisy derivatives. Previous work demonstrated a correlation of 0.8 between joint angles delivered by the Kinect and an optoelectronic system [21]. This could partly explain the low correlation between CRP computed with Kinect and Vicon data. Secondly, there is difficulty in correctly segmenting the feet and the treadmill belt separately during contact phase. This misdetection of the contact point may influence the computation of the shank angle, with a direct consequence on the associated CRP. This hypothesis is supported by the results: CRP based on the shank orientation computed with Kinect had the worst correlation with the one computed using Vicon data. Consequently, designing a new index based on Kinect data such as ILong [16] is of great interest.

In accordance with previous results [16] ILong computed with Kinect data was able to detect asymmetrical gaits across almost all asymmetrical cycles $(\mathrm{H} 2)$. Moreover, the correlation between ILong computed with Vicon and Kinect data was 0.92 . This result has been obtained when placing a $5 \mathrm{~cm}$ sole below one foot to simulate leg length discrepancy, as suggested previously $[16,17,22]$. Further work is required to evaluate the actual sensitivity of the system for a wider set of perturbations to generate new types of asymmetry, such as placing soles of various sizes, or analysing the gait of patients with impaired locomotion.

In previous work the Kinect was placed in front of the subject [16], which is not practicably feasible for some treadmills because of the anteriorly placed console panel. In this paper Kinects were placed both in front and behind the subject to evaluate the potential impact on the validity of this GA index (H3). Results show that both positions have similar validity.

Using a Mean Gait Cycle Model to compute ILong enables the minimisation the impact of noise on the computation of the GA index. However, it requires using several gait cycles, which could be a problem if the gait pattern is not regular or if using a treadmill is not possible. This paper shows that even using a few gait cycles leads to reliable asymmetry index (H4). Even using a unique Kinect gait cycle gives high correlation (0.85) when compared to using reference Vicon data. Although better results could be obtained with more gait cycles, this validity of even a single cycle may be sufficient in most cases. Further 
research would be necessary to accurately estimate the sensitivity of this AG index for various levels of impaired gaits.

In this paper, as suggested previously [16], ILong was computed by selecting a sub-part of the depth image corresponding to the legs, assuming that the legs were within the altitude interval $[0.22 \mathrm{H}-0.47 \mathrm{H}]$, where $\mathrm{H}$ is the total height of the subject. It would also be interesting to evaluate the sensitivity of the method when tuning this interval in each subject's case. Moreover, we could also explore how to deal with the total body for patients with impaired gaits that could be associated with an asymmetric posture of the upper-body. The present protocol has some limitations such as 1) the small number of subjects who participated in the study, 2) only one gait perturbation was tested using a sole below one foot, and 3) recent Kinect correction methods [23] could be used which may improve the CRP.

Despite these shortcomings, determining ILong can be automated. It does not rely upon calibration or markers/sensors placed over the subject. The only constraint in the current version of ILong is use of a treadmill. Based on the results reported in this paper, we could expect that a new version based on a unique gait cycle could be used to study ground locomotion.

\section{Conclusion}

This study shows that CRP computed with skeleton Kinect data failed to assess GA, whereas validity and sensitivity analysis demonstrated reliable assessment when using the new GA index (ILong) based on depth images. This provides the clinician with a longitudinal spacetime GA index within the gait cycle for patients walking on a treadmill. We have shown that placing the depth camera in front of or behind the subject had no strong influence on the results and that even a small number of gait cycles would be enough to detect asymmetry in gait. Further validation of this method is required, using patients with various pathologies to complete the promising results reported above based on a single controlled perturbation. It holds the promise of easy, fast and low-cost within-stride gait longitudinal asymmetry assessment without requiring any specific technical skills. Further development would certainly enable an extension to lateral asymmetry and the possibility to discriminate spatial and temporal asymmetry in gait.

\section{Conflict of interest}

The authors declare that they have no conflict of interest.

\section{Acknowledgements}

This work was funded by the Wellcome Trust and EPSRC grant number 088844/Z/09/Z and the Association nationale de la recherche et de la technologie (ANRT) (France) for the Conventions Industrielles de Formation par la REcherche (CIFRE) 146/2009 funding.

\section{References}

[1] Griffin MP, Olney SJ, McBride ID. Role of symmetry in gait performance of stroke subjects with hemiplegia. Gait\&Posture 1995;3:132-142. 
[2] Böhm H, Döderlein L. Gait asymmetries in children with cerebral palsy: Do they deteriorate with running? Gait\&Posture 2012;35:322-327.

[3] Yogev G, Plotnik M, Peretz C, Giladi N, Hausdorff JM, Gait asymmetry in patients with Parkinson's disease and elderly fallers: when does the bilateral coordination of gait require attention? Exp Brain Res 2007;177:336-346.

[4] James P, Nicol A, Hamblen D. A comparison of gait symmetry and hip movements in the assessment of patients with monoarticular hip arthritis. Clin. Biomech 1994;9:162-166.

[5] Hodt-Billington C, Helbostadb JL, Vervaata W, Rognsvågc T, Moe-Nilssena R. Criteria of gait asymmetry in patients with hip osteoarthritis. Physiotherapy Theory and Practice 2012;28:134-141.

[6] Patterson K K, Gage WH, Brooks D, Black SE, Mcllroy WE. Evaluation of gait symmetry after stroke: A comparison of current methods and recommendations for standardization. Gait\&Posture 2010;31:241-246.

[7] Kaufman KR, Frittoli S, Frigo CA, Gait Asymmetry of Transfemoral Amputees Using Mechanical and Microprocessor-Controlled Prosthetic Knees. Clin Biomech. 2012;27: 460465.

[8] Liao F, Wang J, He P. Multi-resolution entropy analysis of gait symmetry in neurological degenerative diseases and amyotrophic lateral sclerosis, Medical Engineering \& Physics 2008;30:299-310.

[9] Poulet B, de Souza R, Knights CB, Gentry C, Wilson AM, Bevan S, Chang YM Pitsillides AA. Modifications of Gait as Predictors of Natural Osteoarthritis Progression in STR/Ort Mice. Arthritis Rheumatol 2014;66:1832-1842.

[10] Lamb PF, Stöck M. On the use of continuous relative phase: Review of current approaches and outline for a new standard. Clinical Biomechanics 2014;29:484-493.

[11] Haddad JM, van Emmerik REA, Wheat JS, Hamill J, Snapp-Childs W. Relative phase coordination analysis in the assessment of dynamic gait symmetry. J. Appl. Biomech 2010;26:109-113

[12] Combs SA, Dugan EL, Ozimek EN, Curtis AB. Bilateral coordination and gait symmetry after body-weight supported treadmill training for persons with chronic stroke. Clinical Biomechanics 2013;28:448-453.

[13] Clark RA, Bower KJ, Mentiplay BF, Paterson K, Pua YH. Concurrent validity of the Microsoft Kinect for assessment of spatiotemporal gait variables. J. Biomech. 2013;46:27222725.

[14] Springer S, Yogev Seligmann G. Validity of the Kinect for Gait Assessment: A Focused Review. Sensor 2016;16:194-207. 
[15] Auvinet E, Multon F, Aubin CE, Meunier J, Raison M. Detection of gait cycles in treadmill walking using a Kinect. Gait\&Posture 2015;41:722-725.

[16] Auvinet E, Multon F, Meunier J. New Lower-Limb Gait Asymmetry Indices Based on a Depth Camera. Sensors 2015;15:4605-4623.

[17] Gouwanda D, Senanayake SA. Identifying gait asymmetry using gyroscopes-A crosscorrelation and Normalized Symmetry Index approach. J. Biomech. 2011;44:972-978.

[18] Holt KG, Hamill J, Andres RO. Predicting the minimal energy costs of human walking. Med. Sci. Sports Exerc 1991;23:491-498.

[19] Bhave A, Paley D, Herzenberg JE. Improvement in gait parameters after lengthening for the treatment of limb-length discrepancy. J. Bone Joint Surg. Am. 1999;81:529-534.

[20] Leardini A, Cappozzo A, Catani F, Toksvig-Larsen S, Petitto A, Sforza V, Cassanelli G, Giannini S. Validation of a functional method for the estimation of hip joint center location. J. Biomech. 1999;32:99-103.

[21] Pfister A, West AM, Bronner S, Noah JA. Comparative abilities of Microsoft Kinect and Vicon 3D motion capture for gait analysis. J. Med. Eng. Technol. 2014;38:274-280.

[22] Gurney B. Leg length discrepancy. Gait\&Posture 2002;15:195-206.

[23] Plantard P, Shum H, Multon F. Filtered Pose Graph for Efficient Kinect Pose Reconstruction. Multimedia Tools and Applications. 2016 (in Press). 


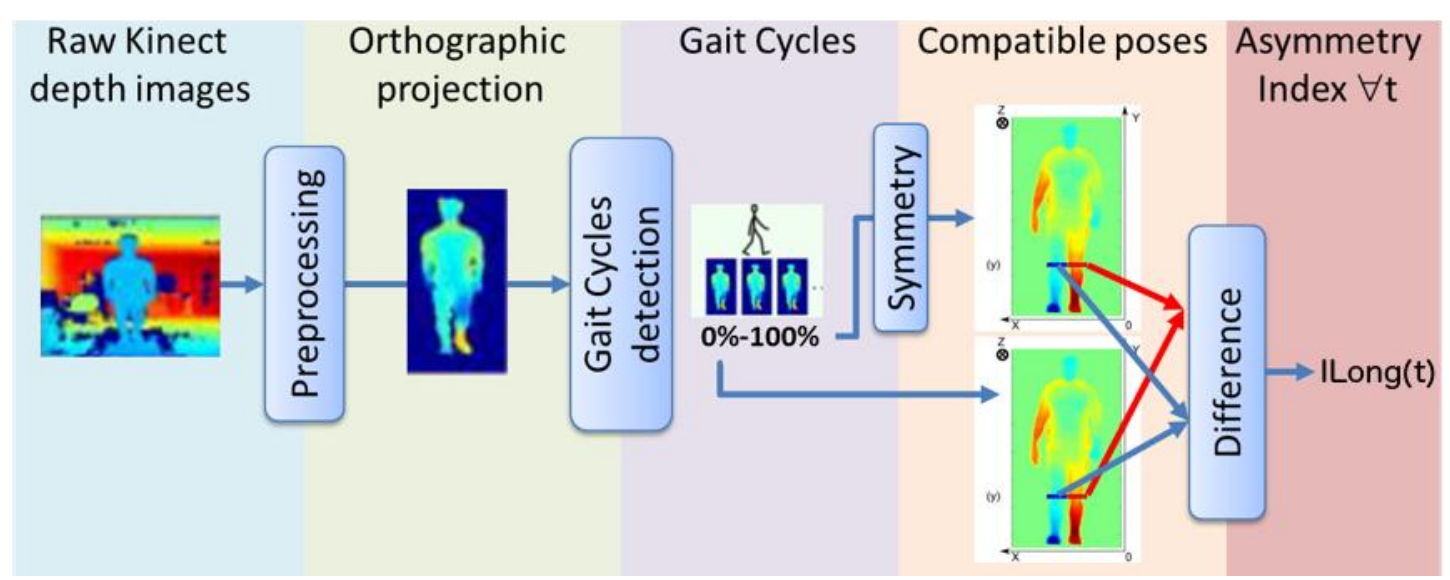

Figure 1: Overview of Kinect data processing to compute the asymmetry index ILong(t). 

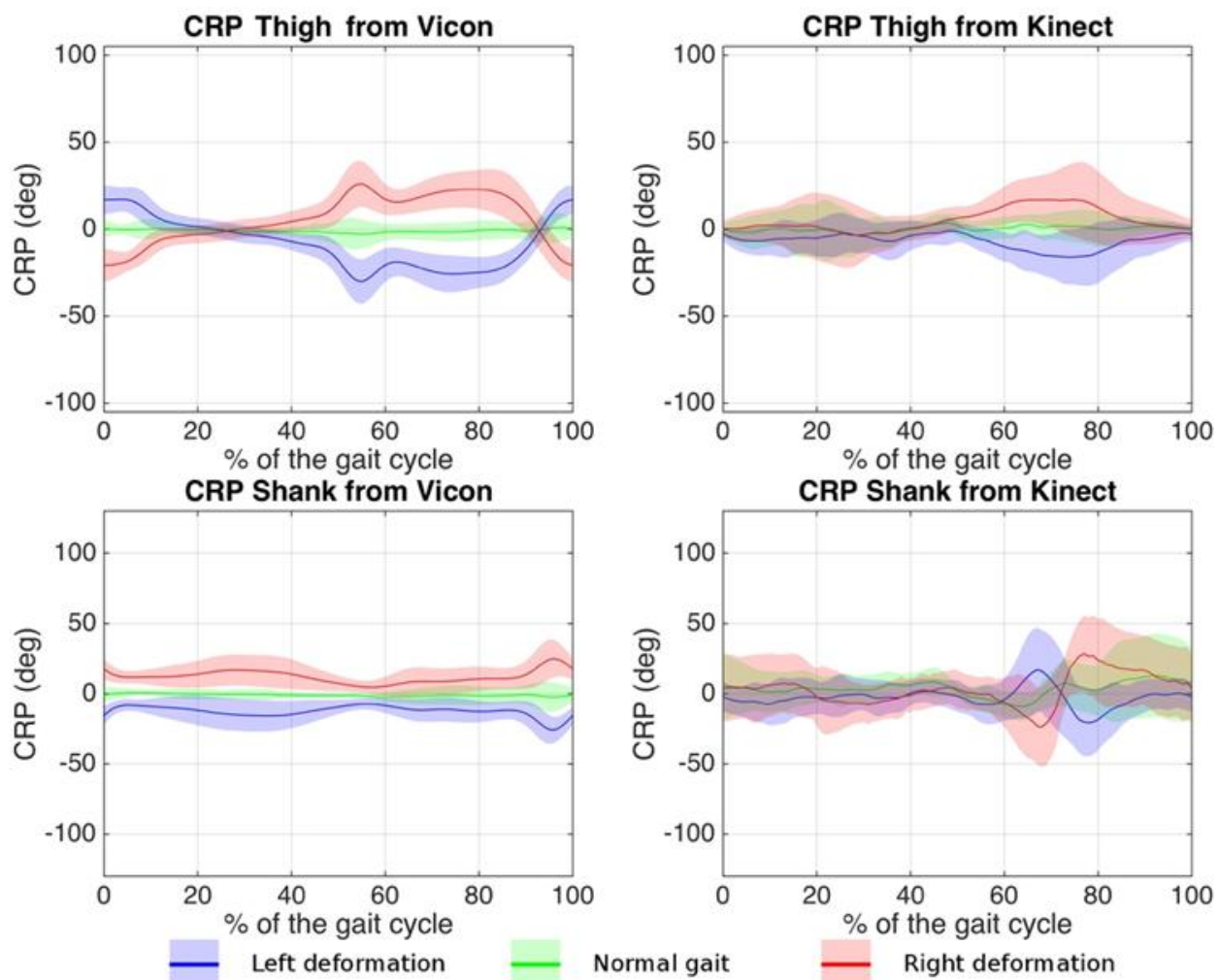

Figure 2: Constant Relative Phase for normal (green) and deformed gait (blue for left deformation and red for right deformation), computed with 90 strides. 

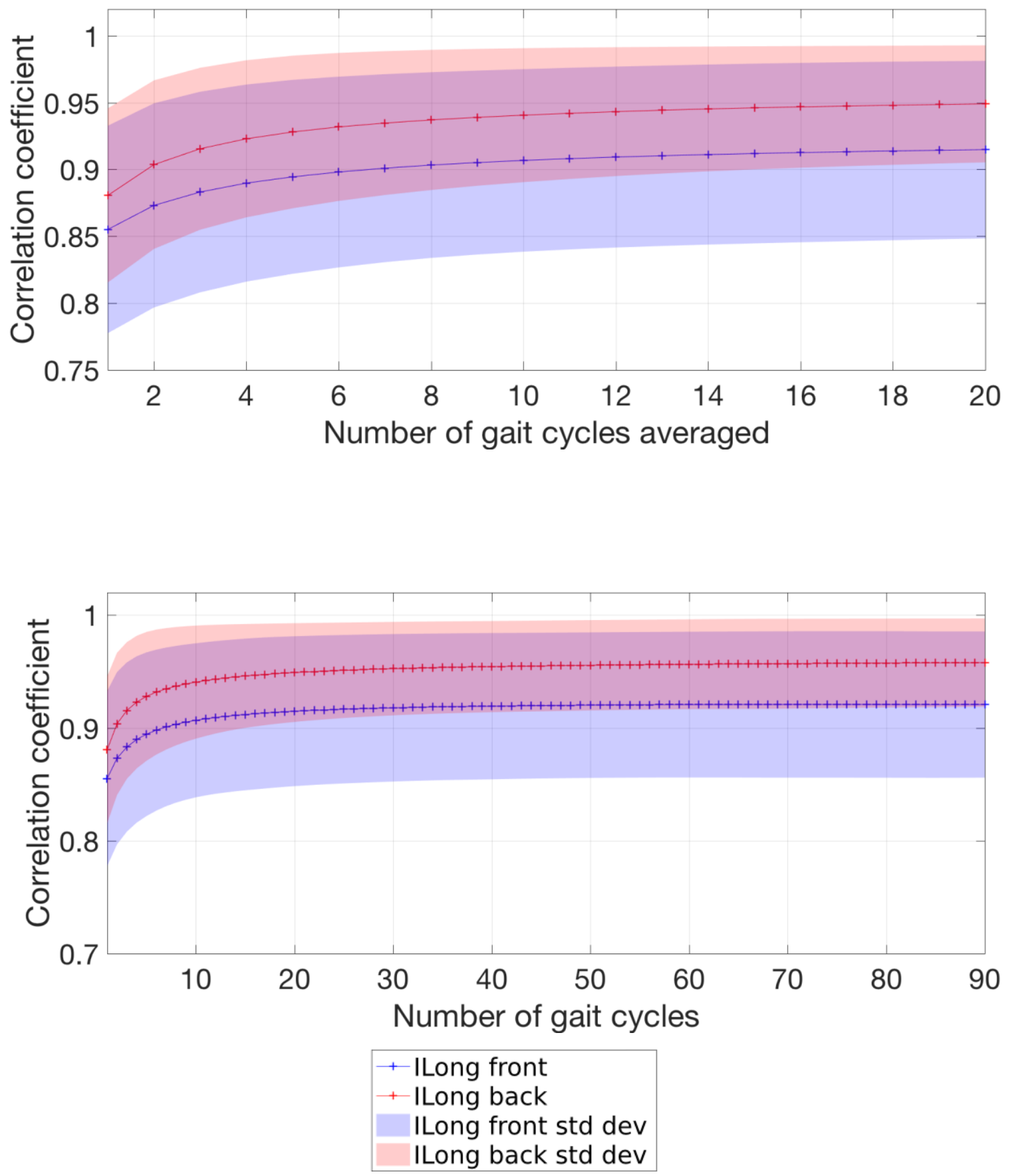

Figure 3: Correlation between ILong computed with Kinect data compared to Vicon data using the same number of gait cycles. Above using a subset of cycles 1 to 90 and below a close up looking at cycles 1 to 20 only. 

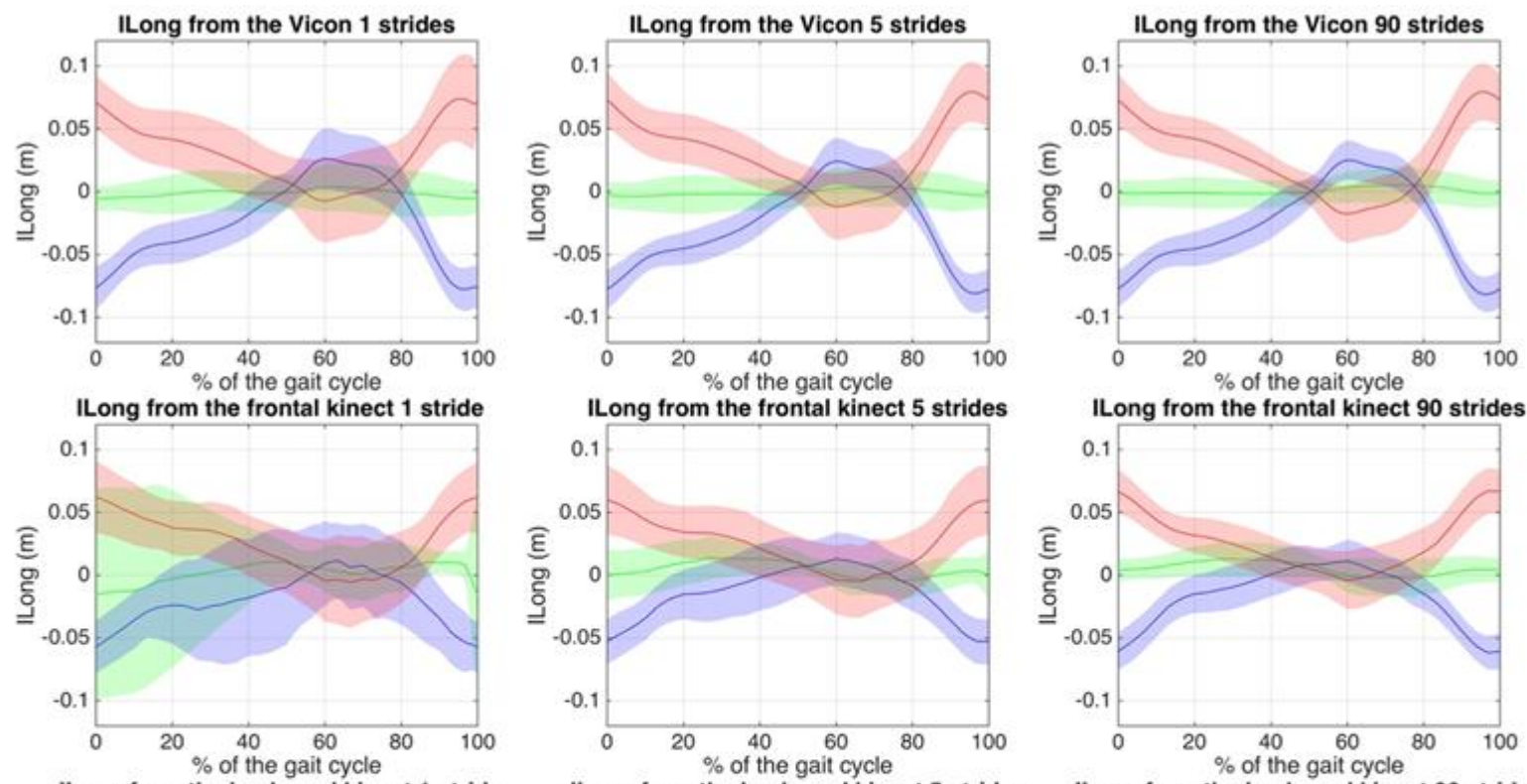

ILong from the backward kinect 1 stride

ILong from the backward kinect 5 strides

ILong from the backward kinect 90 strides
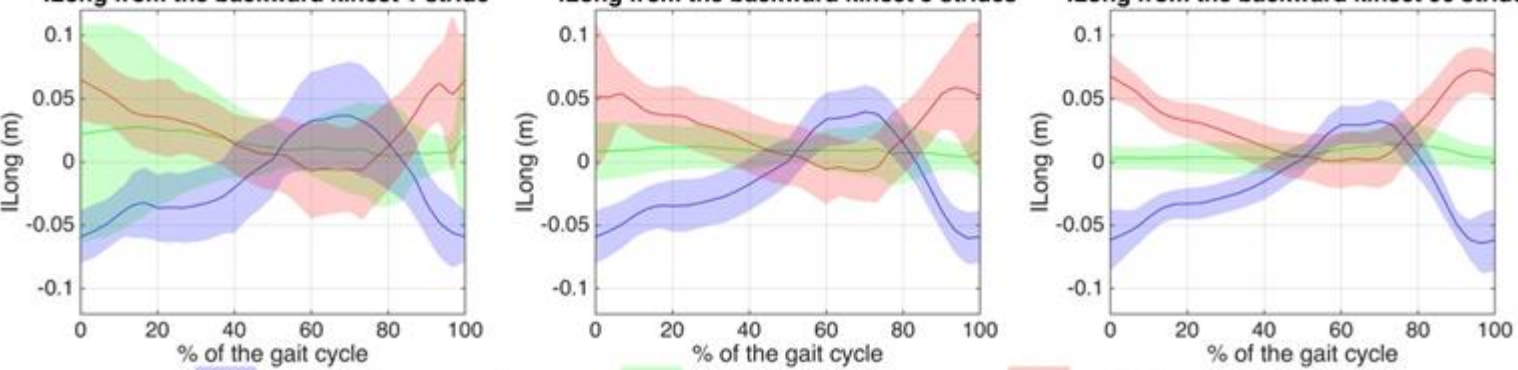

Left deformation

Normal gait

Right deformation

Figure 4: Longitudinal asymmetry index (ILong) for normal (green) and deformed gait (blue for left deformation and red for right deformation), computed for 1, 5 and 90 strides. 
Table 1:

(a) Percent of the stride duration where asymmetry has been detected $(p<0.05)$ with the CRP computed with the Vicon and the Kinect placed in front of the subject.

\begin{tabular}{|c|c|c|}
\hline Measurement & \multicolumn{2}{|c|}{ CRP at shank } \\
\hline Side of deformation & Left & Right \\
\hline Vicon & $100 \%$ & $95 \%$ \\
\hline Kinect & $73 \%$ & $52 \%$ \\
\hline Measurement & $94 \%$ & $95 \%$ \\
\hline Vicon & $77 \%$ & $44 \%$ \\
\hline Kinect & CRP at thigh \\
\hline
\end{tabular}

(b) Percent of the stride duration where asymmetry has been detected (statistical difference $p<0.05$ between asymmetrical and normal trials) using ILong computed with the Vicon, the Kinect placed in front of or behind the subject.

\begin{tabular}{|c|c|c|c|c|c|c|}
\hline Measurement & \multicolumn{6}{|c|}{ ILong } \\
\hline $\begin{array}{c}\text { Number of } \\
\text { strides }\end{array}$ & \multicolumn{2}{|c|}{1} & \multicolumn{2}{|c|}{5} & \multicolumn{2}{|c|}{90} \\
\hline $\begin{array}{l}\text { Side of the } \\
\text { deformation }\end{array}$ & Left & Right & Left & Right & Left & Right \\
\hline Vicon & $56 \%$ & $64 \%$ & $87 \%$ & $86 \%$ & $96 \%$ & $96 \%$ \\
\hline Kinect in front & $16 \%$ & $32 \%$ & $69 \%$ & $59 \%$ & $91 \%$ & $87 \%$ \\
\hline Kinect behind & $56 \%$ & $14 \%$ & $90 \%$ & $74 \%$ & $96 \%$ & $92 \%$ \\
\hline
\end{tabular}

